\title{
El laboratorio en la vigilancia, el control y la investigación de la rabia en España. La experiencia del Centro Nacional de Microbiología
}

\author{
Juan E. Echevarría 1, *, José Miguel Berciano ${ }^{1}$, Ana M. Gavilán García 1, ${ }^{2}$ Joao Carlos Batista Liz ${ }^{1}$ \\ y Aurora Fernández García 1,3 \\ 1 Centro Nacional de Microbiología, Instituto de salud Carlos III, Centro de Investigación Biológica en Red \\ de Epidemiología y Salud Pública (CIBERESP), Madrid; \\ 2 ORCID id: https://orcid.org/0000-0002-6624-2665 \\ 3 ORCID id: https://orcid.org/0000-0002-7504-5321 \\ * Autor correspondencia: jeecheva@isciii.es; ORCID id: https://orcid.org/0000-0001-7522-850X
}

DOI: https://doi.org/10.37536/RIECS.2021.6.1.264

Recibido: 3/05/2021; Aceptado: 20/05/2021; Publicado: 31/05/2021

Resumen: España presenta una situación peculiar Europa, ya que nunca se vio afectada por la epizootia europea de rabia vulpina, pero mantiene un tráfico intenso de bienes y personas con territorios altamente endémicos del norte de África, e incluso fronteras terrestres en Ceuta y Melilla, lo que nos coloca en una situación vulnerable en cuanto a importación de casos. Por otra parte, sabemos de la presencia de dos de los lisavirus de murciélago europeos en nuestro territorio. El laboratorio es un elemento esencial en la vigilancia que se hace imprescindible ante esta situación. El Centro Nacional de Microbiología lleva más de treinta años actuando de laboratorio nacional de referencia en apoyo de los laboratorios autonómicos existentes y haciendo diagnóstico para el resto del territorio, además de representar a España en la red europea de laboratorios RABNET. Así, en el CNM se han diagnosticado y caracterizado la gran mayoría de los casos declarados en España en este período, tanto de rabia de murciélagos, como de rabia importada animal y humana. De manera complementaria a esta actividad de vigilancia, se realizan investigaciones tanto sobre rabia canina como de murciélagos, que, desde hace años, se han ampliado a otros agentes víricos asociados a murciélagos.

Palabras Clave: Rabia, Lisavirus, Murciélagos, Vigilancia, Laboratorio, Enfermedades emergentes.

Abstract: Spain shows peculiarities regarding rabies in Europe. It was not affected by the European vulpine epizootic but keeps an intense traffic of goods and people with highly endemic territories in North Africa and even has terrestrial boundaries in the cities of Ceuta and Melilla. This makes Spain particularly vulnerable to importation of rabies cases. On the other hand, we know the presence in Spain of two of the five bat lyssaviruses endemic from Europe. The laboratory is an essential tool for the surveillance that is strictly necessary to keep in order to handle this situation. The National Center of Microbiology (CNM) provides support to autonomic laboratories, makes primary diagnosis for the rest of the territory and represents Spain in the European laboratory net RABNET from more than thirty years to now, according to its role of National Reference Laboratory. During this period, the CNM has diagnosed and characterized most rabies cases declared in Spain, including both bat rabies and imported animal and human cases. Associated to these surveillance activities, the CNM makes research in the field of canine and bat rabies, which has been extended to other bat-associated viruses in the last years.

Key words: Rabies, Lyssavirus, Bats, Surveillance, Laboratory, Emerging diseases. 
La rabia es una enfermedad temida por la humanidad desde tiempos antiguos a causa de las dramáticas características de sus síntomas y su implacable letalidad. Los médicos griegos y romanos nos dejaron ya descripciones clínicas precisas de una enfermedad que asociaban claramente a la mordedura de los perros (King et al. 2004). Sin embargo, algunos textos legales de antiguas civilizaciones babilónicas ya regulan las indemnizaciones que se debían satisfacer por los daños causados por los daños producidos por los perros "locos", lo que se ha interpretado como una posible referencia anterior a la enfermedad.

La rabia está en los mismos orígenes de la Microbiología y la lucha contra las enfermedades infecciosas. En el año 1885 Louis Pasteur administraba el primer tratamiento de inmunoprofiláxis post exposición, demostrando la eficacia de una vacuna que más adelante se convertiría en la base del control de la rabia canina y a la postre de la humana. El moderno concepto "one health" de una Salud humana y veterinaria que han de ir necesariamente de la mano es, pues, una aproximación clásica cuando hablamos de la rabia. En 1904, el patólogo italiano Adelchi Negri publicaba sus hallazgos patológicos en el cerebro de los perros rabiosos y describía sus famosos corpúsculos, proporcionando un método de diagnóstico de laboratorio que estaría vigente durante décadas. La patóloga estadounidense Anna Wessels Williams obtuvo hallazgos similares pero no los publicó con la celeridad que lo hizo Negri, habiendo quedada eclipsada para la historia de la ciencia. A principios del siglo XX estaban, pues, disponibles todas las herramientas básicas para la vigilancia y el control de la rabia. Sin embargo, más de cien años después, siguen muriendo alrededor de 59.000 personas de rabia cada año (Hampson, 2015), la mayoría de ellos niños. ¿Cómo es esto posible? Un simple vistazo sobre el mapa de distribución de la rabia humana nos da la respuesta (Fahrion, 2016). Las zonas de mayor endemia se encuentran en el África subsahariana y en el sur de Asia y los países más afectados están entre los más desfavorecidos del planeta. Dentro de ellos, las zonas rurales más remotas son las más afectadas. Las razones de estas muertes no son, pues de índole técnico, sino socio-económico y político, por esta razón la rabia está dentro de la lista de enfermedades olvidadas de la OMS y la comunidad internacional se ha planteado como objetivo eliminar la rabia humana transmitida por el perro para el año 2030 (WHO, 2018). Parece un objetivo muy ambicioso, pero un breve recorrido por la historia de la lucha contra la rabia en Europa nos mostrará que cuando se disponen los medios adecuados, es perfectamente posible esto y mucho más.

\section{Rabia en Europa o la evidencia de que casi todo es posible}

Europa era endémica para rabia canina a principios del siglo XX. El control de las poblaciones caninas y la introducción de las nuevas herramientas aportadas por la Ciencia, permitió ir eliminándola de la mayoría del continente europeo a lo largo del siglo XX. Sin embargo, a mediados de los años 40, coincidiendo con el final de la segunda guerra mundial, se declaró una epizootia en zorros, cuyo origen geográfico se sitúa en la frontera ruso-polaca. La epizootia vulpina europea fue extendiéndose como una mancha de aceite durante las décadas siguientes, ocupando toda Centroeuropa a mediados de los años 90, de manera que su entrada en la Península Ibérica solo parecía cuestión de tiempo (King, 2004). Muy al contrario, la implementación de campañas de vacunación vulpinas mediante vacunas de virus de la rabia (RABV) atenuado dispuestas en cebos dispersados por el campo, ha ido barriendo la epizootia hasta situarla a día de hoy fuera del territorio de la Unión Europea (Figura 1), que actualmente mantiene campañas a lo largo de su frontera este y ha comenzado a colaborar con los países fronterizos para su control. De esta manera, la UE solo declara actualmente casos aislados o brotes autolimitados de rabia vulpina en áreas fronterizas con países extracomunitarios endémicos. Fuera de estos territorios solo se declaran casos importados por tres vías fundamentales. La más frecuente (aproximadamente la mitad de los casos) tiene su origen en Marruecos, casi siempre a través de la adopción de cachorros de perro por turistas, que los introducen escondidos a través de las fronteras españolas para desplazarse en automóvil hasta el país de destino, generalmente Francia (Mailles, 2011). Otra vía menos frecuente sería la importación de perros infectados por carretera desde países del este de Europa endémicos para rabia vulpina y finalmente la importación por vía aérea desde países endémicos de Asia o África. El último caso autóctono en la UE se produjo en Rumanía, en el año 2012. Entre los años 2006 y 2019 se han 
producido un total de 18 casos importados de rabia humana en la UE y el área económica europea. En todos los casos, se trató de viajeros que adquirieron la enfermedad en países endémicos, siendo la India (5 casos) y Marruecos (4 casos) los más frecuentemente implicados (Gautret, 2011; Gossner, 2020).

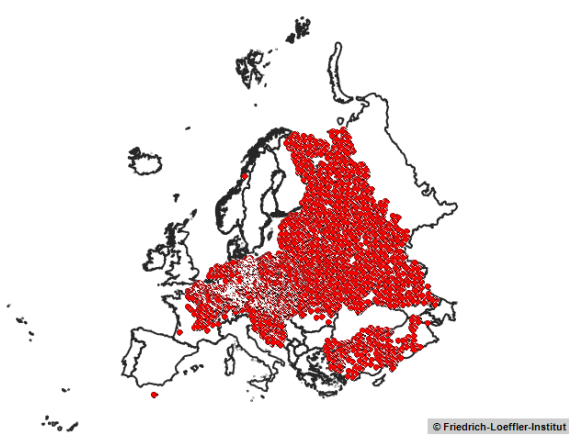

1990

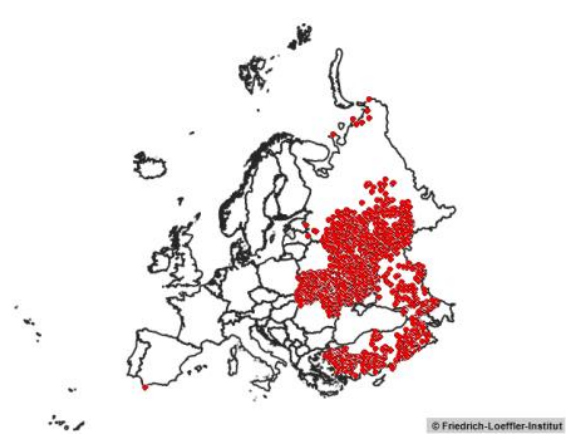

2019

Figura 1. Situación de la rabia en Europa en 1994 (izquierda) y 2019 (derecha). Fuente: Rabies Bulletin Europe (www.who-rabies-bulletin.org). Centro colaborador de la OMS para investigación y vigilancia de la rabia. Instituto de Virología Molecular y Biología Celular, Instituto Friedrich-Loeffler, Isla de Riems, Alemania.

El único continente donde los murciélagos son reservorios de RABV es América. Además, en su franja tropical y subtropical existen murciélagos hematófagos, comúnmente conocidos como vampiros, que, a diferencia del resto de las especies de murciélagos, no muerden a los humanos y otros animales de manera fortuita, sino como parte de su comportamiento trófico. Solo tres de las más de 1.400 especies de murciélagos que existen en el mundo son hematófagas y las tres viven en el continente americano. Esta combinación de un virus particularmente adaptado a infectar un amplio rango de mamíferos y de la existencia de huéspedes con comportamientos particularmente proclives a la transmisión del virus, hace que la importancia de la rabia transmitida por murciélagos en América no sea comparable con la del resto el mundo, donde no hay especies hematófagas y los lisavirus que les infectan son aparentemente menos transmisibles. En el momento actual se conocen otros diecisiete lisavirus, aparte de RABV (Tabla I), quince de los cuales tienen a los murciélagos como hospedadores. Cada uno de ellos es específico de un área geográfica reducida y está asociado a especies diferentes de murciélagos. La problemática de la rabia en murciélagos es, pues, totalmente diferente en cada continente. Mientras que en América se han descrito cientos de casos rabia humana transmitida por murciélagos, en el resto del mundo solo se han contabilizado once (Johnson, 2010), cinco de los cuales han ocurrido en Europa, tres por lisavirus europeo de murciélago tipo 1 (EBLV-1) y dos por lisavirus europeo de murciélago tipo 2 (EBLV-2) (Johnson, 2010; Regnault, 2021). El primero de ellos se declaró en 1986 en Finlandia. Investigaciones retrospectivas realizadas en otros territorios con motivo de la declaración de este caso desvelaron otros dos (Rusia 1985 y Ucrania 1977). Estos hallazgos motivaron el desarrollo de sistemas de vigilancia y de protocolos de prevención basados en la educación sanitaria de la población, la profilaxis post-exposición y la vacunación preventiva de profesionales expuestos a murciélagos. Así, entre 1977 y 1984 tan solo se habían declarado dos casos de rabia en murciélagos, mientras que a partir de ese momento hasta finales de 2020 se han comunicado 1.319. De aquellos con datos disponibles de especie de hospedador y virus, el $95 \%$ corresponden a EBLV-1 en Eptesicus serotinus y Eptesicus isabellinus, unas pocas decenas a EBLV-2 en Myotis daubentonii y Myotis dasycneme, siendo esporádicos los casos por el resto de lisavirus europeos (Schatz, 2013). Desde la entrada en vigor de los protocolos preventivos, solo se han comunicado dos casos humanos, uno en un voluntario de un centro de recuperación de fauna salvaje en Escocia y otro recientemente en una persona que no presentaba prácticas de riesgo (Nathwani, 2003; Regnault, 2021). Ambos casos podían haberse evitado mediante profilaxis post-exposición de haber acudido al médico tras la mordedura y el primero, además, debería de haber estado vacunado preventivamente antes de manejar murciélagos, muy especialmente tratándose de ejemplares enfermos. 
Tabla I Especies del género Lyssavirus

\begin{tabular}{|c|c|c|c|c|}
\hline Lisavirus & $\begin{array}{l}\text { ¿Ofrece } \\
\text { protección } \\
\text { la vacuna? }\end{array}$ & $\begin{array}{c}\text { Casos } \\
\text { humanos } \\
\text { asociados }\end{array}$ & $\begin{array}{l}\text { Especies de } \\
\text { asociadas con } \\
\text { certeza*. (en } \\
\text { negrita las } \\
\text { existentes en } \\
\text { España) }\end{array}$ & $\begin{array}{l}\text { Paises donde se han } \\
\text { detectado. }\end{array}$ \\
\hline $\begin{array}{l}\text { Aravan } \\
\text { lyssavirus } \\
\text { (ARAV) } \\
\end{array}$ & Sí & No & Myotis blythi & Kyrgystan \\
\hline $\begin{array}{l}\text { Australian } \\
\text { bat } \\
\text { lyssavirus } \\
\text { (ABLV) }\end{array}$ & Sí & Sí (3) & $\begin{array}{l}\text { Pteropus alecto } \\
\text { Pteropus } \\
\text { scapulatus } \\
\text { Pteropus } \\
\text { poliocephalus } \\
\text { Pteropus } \\
\text { conspicillatus } \\
\text { Saccolaimus } \\
\text { flaviventris }\end{array}$ & Australia \\
\hline $\begin{array}{l}\text { Bokeloh } \\
\text { bat } \\
\text { lyssavirus } \\
\text { (BBLV) }\end{array}$ & Sí & No & $\begin{array}{l}\text { Complejo } \\
\text { Myotis } \\
\text { nattereri }\end{array}$ & $\begin{array}{l}\text { Alemania, Francia, } \\
\text { Polonia }\end{array}$ \\
\hline $\begin{array}{l}\text { Duvenhage } \\
\text { lyssavirus } \\
\text { (DUVV) }\end{array}$ & Sí & Sí (3) & $\begin{array}{l}\text { Miniopterus sp. } \\
\text { Nycteris } \\
\text { thebaica }\end{array}$ & $\begin{array}{l}\text { Sudáfrica, } \\
\text { Kenia } \\
\text { Zimbabwe }\end{array}$ \\
\hline $\begin{array}{l}\text { European } \\
\text { bat } 1 \\
\text { Lyssavirus } \\
\text { (EBLV-1) }\end{array}$ & Sí & Sí (3) & $\begin{array}{l}\begin{array}{l}\text { Eptesicus } \\
\text { serotinus } \\
\text { (cientos de casos) }\end{array} \\
\text { Eptesicus } \\
\text { isabellinus } \\
\text { (decenas de } \\
\text { casos. Sur de } \\
\text { España.) } \\
\text { Casos } \\
\text { esporádicos en: } \\
\text { Vespertilio } \\
\text { murinus, } \\
\text { Pipistrellus } \\
\text { nathusii, oveja, } \\
\text { garduña, gato. } \\
\text { Pipistrellus } \\
\text { pipistrellus (un } \\
\text { solo caso) }\end{array}$ & $\begin{array}{lr}\text { Francia, } & \text { Alemania, } \\
\text { Holanda, } & \text { Polonia, } \\
\text { Dinamarca, } & \text { España, } \\
\text { Ucrania, } & \text { Rusia, } \\
\text { Hungría, } & \text { Bélgica, } \\
\text { Luxemburgo, } & \text { Reino } \\
\text { Unido. }\end{array}$ \\
\hline $\begin{array}{l}\text { European } \\
\text { bat } 2 \\
\text { lyssavirus } \\
\text { (EBLV-2) }\end{array}$ & Sí & Sí (2) & $\begin{array}{l}\text { Myotis } \\
\text { daubentonii } \\
\text { Myotis } \\
\text { dasycneme }\end{array}$ & $\begin{array}{l}\text { Holanda, Suiza, Reino } \\
\text { Unido, Alemania, } \\
\text { Finlandia, Noruega, } \\
\text { Dinamarca }\end{array}$ \\
\hline $\begin{array}{c}\text { Gannoruwa } \\
\text { bat }\end{array}$ & Sí & No & Pteropus medius & Sri Lanka \\
\hline
\end{tabular}




\begin{tabular}{|c|c|c|c|c|}
\hline $\begin{array}{c}\text { lyssavirus } \\
\text { (GBLV) }\end{array}$ & & & & \\
\hline $\begin{array}{c}\text { Ikoma } \\
\text { lyssavirus } \\
(\mathrm{IKOV}) \\
\end{array}$ & No & No & $\begin{array}{l}\text { No asociado a } \\
\text { murciélagos. } \\
\text { Civeta. }\end{array}$ & Tanzania \\
\hline $\begin{array}{c}\text { Irkut } \\
\text { lyssavirus } \\
\text { (IRKV) }\end{array}$ & Sí & Sí (1) & $\begin{array}{l}\text { Murina } \\
\text { leucogaster }\end{array}$ & Federación Rusa, China \\
\hline $\begin{array}{l}\text { Kotolahti } \\
\text { Bat } \\
\text { Lyssavirus } \\
\text { (KBLV)\$* } \\
\end{array}$ & Sí & No & Myotis brandtii & Finlandia \\
\hline $\begin{array}{l}\text { Khujand } \\
\text { lyssavirus } \\
\text { (KHUV) }\end{array}$ & Sí & No & $\begin{array}{l}\text { Myotis } \\
\text { mystacinus }\end{array}$ & Tajikistan \\
\hline $\begin{array}{l}\text { Lagos bat } \\
\text { lyssavirus } \\
(\mathrm{LBV})\end{array}$ & No & No & $\begin{array}{l}\text { Eidolon helvum } \\
\text { Rousettus } \\
\text { aegyptiacus } \\
\text { Micropteropus } \\
\text { pussilus } \\
\text { Nycteris } \\
\text { gambiensis } \\
\text { Epomophorus } \\
\text { wahlbergi }\end{array}$ & $\begin{array}{l}\text { Nigeria, } \\
\text { Senegal, } \\
\text { Ghana, } \\
\text { Guinea } \\
\text { Kenia } \\
\text { Francia (ex-Togo o } \\
\text { Egipto), } \\
\text { República } \\
\text { Centroafricana } \\
\text { Sudáfrica }\end{array}$ \\
\hline $\begin{array}{c}\text { Lleida bat } \\
\text { lyssavirus } \\
\text { (LLEBV) }\end{array}$ & No & No & $\begin{array}{l}\text { Miniopterus } \\
\text { schreibersii }\end{array}$ & España, Francia \\
\hline $\begin{array}{l}\text { Mokola } \\
\text { Lyssavirus } \\
\text { (MOKV) }\end{array}$ & No & Sí (2) & $\begin{array}{l}\text { No asociado a } \\
\text { murciálegos. } \\
\text { Perro, gato, } \\
\text { musaraña. }\end{array}$ & $\begin{array}{l}\text { Nigeria, Camerún, } \\
\text { Sudáfrica, República } \\
\text { Centroafricana, } \\
\text { Zimbawe, Etiopía }\end{array}$ \\
\hline $\begin{array}{l}\text { Rabies } \\
\text { lyssavirus } \\
(\mathrm{RABV})^{\circ}\end{array}$ & Sí & $\begin{array}{l}\text { Sí } \\
\text { (60.000/año) }\end{array}$ & $\begin{array}{l}\text { Órdenes } \\
\text { Carnivora, y } \\
\text { Quiroptera (solo } \\
\text { en América). }\end{array}$ & $\begin{array}{l}\text { Todo el mundo salvo } \\
\text { Australia, la Antártida } \\
\text { y algunos territorios } \\
\text { insulares. Eliminado de } \\
\text { gran parte de Europa. }\end{array}$ \\
\hline $\begin{array}{l}\text { Shimoni } \\
\text { bat } \\
\text { lyssavirus } \\
\text { (SHIBV) }\end{array}$ & No & No & $\begin{array}{l}\text { Hipposideros } \\
\text { commersoni }\end{array}$ & Kenia \\
\hline $\begin{array}{l}\text { Taiwan bat } \\
\text { lyssavirus } \\
(\mathrm{TWBLV}) \$ \\
\end{array}$ & $\begin{array}{c}\text { Sí ( sin } \\
\text { constancia } \\
\text { empírica) }\end{array}$ & No & $\begin{array}{l}\text { Pipistrellus } \\
\text { abramus }\end{array}$ & Taiwan \\
\hline $\begin{array}{c}\text { West } \\
\text { Caucasian } \\
\text { bat } \\
\text { lyssavirus } \\
\text { (WCBV) }\end{array}$ & No & No & $\begin{array}{l}\text { Miniopterus } \\
\text { schreibersii. } \\
\text { Gato. }\end{array}$ & Federación Rusa, Italia. \\
\hline
\end{tabular}




\section{La rabia en España y sus peculiaridades}

España ha sido un país endémico para rabia canina hasta que se eliminó en 1965, en lo que supone una de nuestras mayores epopeyas en Salud Pública. Sin embargo, diez años después, se declaraba un brote en la provincia de Málaga, afectando a más de 120 animales entre 1975 y 1978 y demostrando la necesidad de no bajar la guardia y establecer buenos sistemas de vigilancia. Desde entonces solo hemos tenido casos importados con regularidad en las ciudades autónomas de Ceuta y Melilla, que son difíciles de evitar dadas las especiales características de sus fronteras terrestres con territorios endémicos de Marruecos (King 2004, Mingo 2017). La elevada cobertura vacunal existente en la población canina de estas ciudades, que son las únicas en las que está sufragada por el Estado, previene la aparición de brotes derivados de estos episodios recurrentes de importación. Como antes indicábamos, tenemos evidencias del tránsito por territorio peninsular de animales infectados recogidos en Marruecos y ocultados durante el paso por la frontera, que han acabado produciendo alertas en los países de destino una vez alcanzada la fase clínica de la enfermedad. En junio de 2013 ocurrió lo que parecía inevitable y se diagnosticaba un caso de rabia en un perro abatido por la policía en Toledo tras causar graves lesiones a varias personas. Sus propietarios españoles, había viajado con él a Marruecos tras la administración de vacuna, pero sin cumplir los plazos reglamentarios para que pudiese hacer su efecto, ni haber comprobado con un ensayo de anticuerpos la inmunización efectiva del animal, tal y como prescriben las normas internacionales para movimientos transfronterizos de animales a países endémicos de rabia. El animal contrajo la enfermedad en Marruecos y tras varios intentos infructuosos, los dueños lograron reintroducirlo ilegalmente en España, donde, al cabo de varias semanas desarrolló síntomas. La investigación epidemiológica del caso reveló, igualmente, que, antes de ser abatido, había estado perdido durante 24 horas con claros síntomas clínicos y se había desplazado $10 \mathrm{Km}$. Durante el período previo a los síntomas en el que se considera que el animal puede ser contagioso, había transitado con sus dueños por varias localidades de otras dos comunidades autónomas diferentes. Esta alerta epidemiológica supuso la prohibición durante seis meses de los movimientos transfronterizos de animales, la designación de varias zonas de riesgo con limitación absoluta de movimientos internos de animales, la administración de cientos de tratamientos de inmunoprofiláxis y de observaciones domiciliarias de animales y, en definitiva, la movilización de un importante contingente de profesionales sanitarios y de cuerpos y fuerzas de seguridad del Estado. Afortunadamente, las medidas de vigilancia y control funcionaron y no se registraron casos secundarios ni hubo que lamentar víctimas humanas, aunque los daños económicos fueron, desde luego, muy cuantiosos. (Pérez de Diego, 2013; Suárez-Rodríguez 2013)

El último caso autóctono de rabia humana en la Península ocurrió en 1975 durante el brote de Málaga y en 1978 se declaraban los dos últimos en Melilla. Desde entonces solo hemos tenido tres casos importados. En el año 2004 un turista austríaco que había sido mordido por un perro en Marruecos acudía al hospital de Ceuta con síntomas de rabia que se confirmaron en el laboratorio (Strauss, 2004). Posteriormente, en Madrid en 2014 y en Vizcaya en 2019 (Gossner, 2020), se declararon sendos casos en ciudadanos marroquíes que habían sido mordidos por perros en su país y habían viajado a España durante el período de incubación.

En el año 1987 se informaba desde Valencia del primer murciélago con rabia detectado en España. Desde entonces, se han declarado un total de 42 casos. En cuarenta de ellos se encontró lisavirus europeo de murciélago tipo 1 (EBLV-1) en sus especies hospedadoras características: Eptesicus serotinus en la mitad norte de la Península y su especie críptica vicariante en la mitad sur, Eptesicus isabellinus.

España, pues, presenta peculiaridades en cuanto a su situación epidemiológica para rabia con respecto a otros estados miembros de la UE. En primer lugar, nunca fue alcanzada por la epizootia europea de rabia vulpina, además, mantiene fronteras terrestres con territorios norteafricanos altamente endémicos para rabia canina y un intenso tránsito de vehículos a través del Estrecho de Gibraltar que han servido de medio para la entrada irregular de animales infectados, causando una alerta en España y varias en Francia. Finalmente, es el único país con dos especies diferentes de murciélagos reservorias de EBLV-1, una de las cuales se distribuye también por el Norte de África. 
Todo ello hace de la vigilancia de la rabia en España una actividad de ámbito internacional que debe realizarse de acuerdo a nuestras peculiaridades (Mingo, 2017).

\section{El Centro Nacional de Microbiología en la vigilancia de la rabia en España}

El brote de rabia de los años 70 en Málaga supuso un impulso importante de las actividades de laboratorio en la vigilancia de la rabia, encargándose de ello la Escuela Nacional de Sanidad, que a la postre sería una de las semillas del futuro Instituto de Salud Carlos III. En el año 1987, la actividad de diagnóstico de laboratorio de rabia se transfiere al Centro Nacional de Microbiología (CNM). En el año 1994, el RD 2491/1994 sobre medidas de protección contra zoonosis y posteriormente en 2004 el RD 1940/2004 sobre la vigilancia de las zoonosis y los agentes zoonóticos, designan al CNM como laboratorio nacional de referencia para los casos sospechosos de zoonosis en el hombre y en animales sospechosos de rabia. En el año 2011, el CNM estructura parte de sus actividades en programas de vigilancia microbiológica, entre el que se encuentra el de rabia animal y humana. La única comunidad autónoma con laboratorio de rabia disponible entre 1987 y el momento actual es la Comunidad Valenciana. Andalucía tuvo laboratorio primario hasta 1999, dando también servicio a Melilla, mientras que Aragón lo tiene desde 2011. Además de ser laboratorio nacional de referencia para estos laboratorios autonómicos, el CNM actúa como laboratorio primario para el resto del territorio del Estado. El CNM está integrado en RABNET, la red de laboratorios de rabia de la UE, cuyo laboratorio de referencia es el ANSES, en Nancy, Francia. Así, el CNM garantiza la disponibilidad en todo el Estado de todas las herramientas de diagnóstico de laboratorio necesarias para orientar los tratamientos de inmunoprofiláxis post-exposición en personas expuestas a animales sospechosos y proporcionar a las autoridades competentes en sanidad humana y veterinaria los datos necesarios para mantener una buena vigilancia epidemiológica y hacer las oportunas notificaciones internacionales.

El diagnóstico en animales sospechosos, o el post-mortem en casos humanos, se realiza sobre el encéfalo (OMS 2018, OIE 2019). De acuerdo a los procedimientos actualmente en vigor en España para la actuación ante mordeduras de animales (Ministerio de Agricultura, 2013) y la legislación vigente, los perros y gatos agresores deben de ser sometidos a un período de observación de 14 días bajo la supervisión de un veterinario oficial quien, si el animal manifiesta síntomas compatibles o muere, ordenará el envío del cerebro al laboratorio para su análisis. Para el resto de los animales no existen criterios fiables en los que basar períodos de observación y se sacrificarán o no para su estudio en función de las circunstancias y riesgos clínico-epidemiológicos.

El diagnóstico ante-mortem de casos humanos por métodos directos se realiza sobre saliva, líquido cefalorraquídeo y biopsia de piel de nuca, siendo esta última muestra la de mejor rendimiento. La detección de anticuerpos específicos en suero permite diagnosticar el caso en ausencia de antecedentes de vacunación.

Las técnicas actualmente disponibles en el CNM son las indicadas en los manuales de laboratorio de la OMS y la OIE (OMS 2018, OIE 2019). Las muestras son procesadas en el laboratorio de bioseguridad de nivel 3 hasta su inactivación y luego se finaliza en nivel 2. Las técnicas empleadas están acreditadas bajo normas ISO 15189 e ISO 17025.

Las peticiones analíticas se realizan de forma remota en una plataforma web previa obtención de credenciales de acceso. Los resultados, una vez validados por el facultativo, pueden ser consultados y descargados en la misma plataforma. Todas las muestras son cribadas por detección de antígenos, PCR a tiempo real múltiple para RABV y EBLV-1 y PCR genérica de lisavirus. En casos de sospecha de rabia humana los sueros se ensayan para anticuerpos específicos por enzimoinmunoanálisis.

La detección de antígenos víricos mediante inmunofluorescencia se realiza sobre dos improntas de diferentes zonas del encéfalo empleando dos reactivos inmunológicos comerciales específicos diferentes, uno policlonal y otro monoclonal. La lectura al microscopio de fluorescencia la realizan dos personas diferentes de manera independiente, teniendo que ser los resultados coincidentes para su emisión (figura 2). 


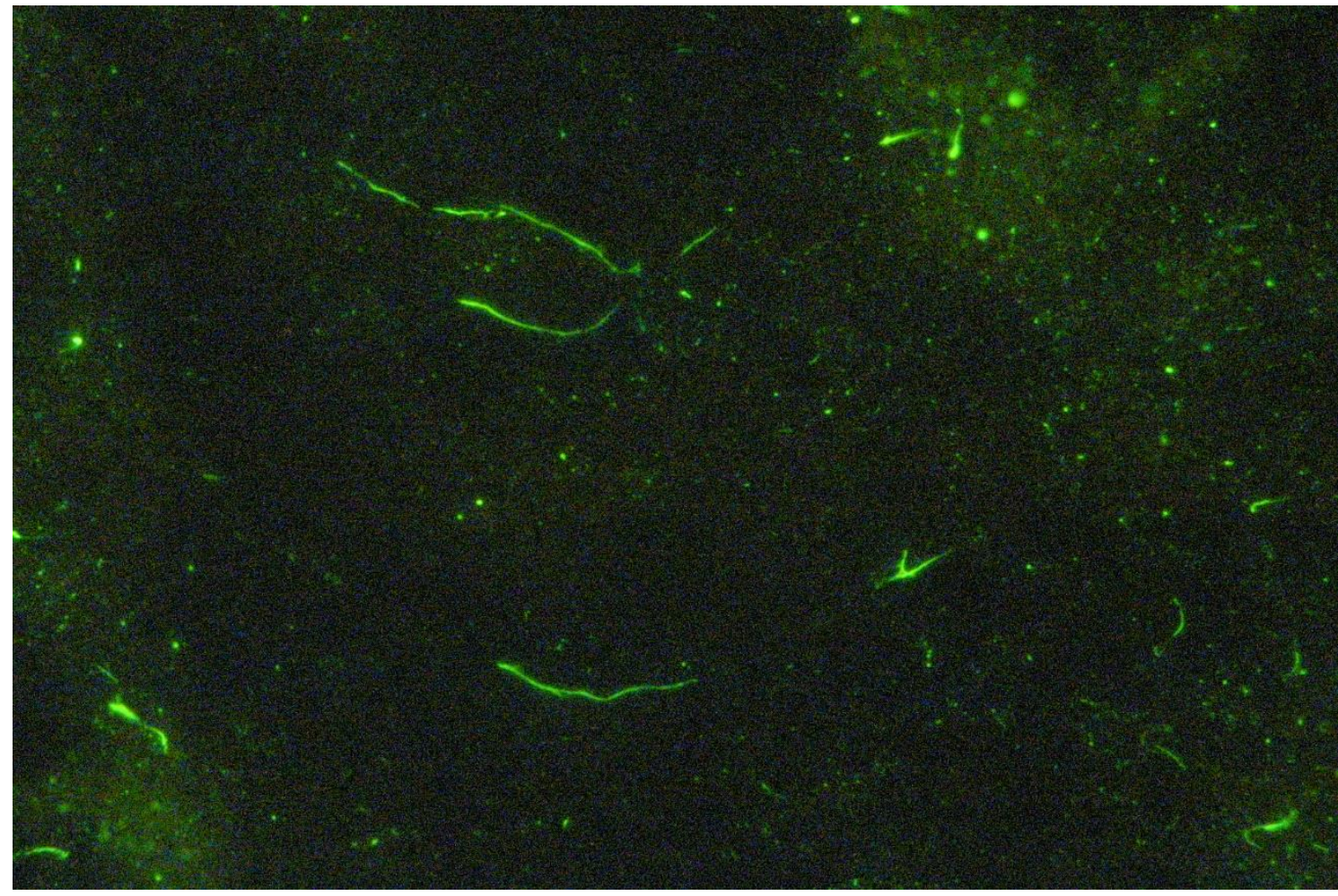

Figura 2 Imagen de detección de antígenos de virus de la rabia por inmunofluorescencia.

La detección de genomas víricos por PCR a tiempo real múltiple de RABV y EBLV-1 es una modificación de un protocolo publicado (Hoffman, 2010) que permite detectar de manera simultánea y específica los dos virus más comúnmente encontrados en España. Esto es importante hacerlo así ya qué, si bien cada uno de los virus tiene un rango de reservorios reducido, se han descrito infecciones aisladas en huéspedes heterólogos ("spill over"), tales como infecciones por EBLV-1 en ovejas (Tjørnehøj, 2006), una garduña (Müller, 2004) o gatos (Dacheux, 2009) que no serían detectables con cribados dirigidos exclusivamente a murciélagos. La PCR genérica de lisavirus es de desarrollo propio (Vázquez, 2006) y permite detectar cualquier miembro del género lisavirus, incluso si no está escrito. El sentido de incluirla sería detectar, tanto casos importados causados por lisavirus inesperados, como el de un murciélago importado infectado por virus Lagos bat (LBV) que se detectó en 1999 en Francia (Picard-Meyer, 2004), o el de un gato infectado por Virus de murciélago del Cáucaso occidental (WCBV) en Italia (comunicación Dra. P. di Benedictis). Asimismo, esta técnica nos permitió detectar un lisavirus desconocido, dando lugar a la descripción original del lisavirus de murciélago Lleida (LLEBV) (Aréchiga-ceballos, 2013).

Cualquier resultado positivo se caracteriza por secuenciación genómica del gen de la nucleoproteína a fin de identificar el virus y en su caso el subtipo o la variante geográfica (VázquezMorón, 2011). Cuando el animal analizado tiene dueño, su procedencia se puede conocer a través de una encuesta epidemiológica, pero, en caso contrario, la secuenciación sería la única manera de poder trazar el origen geográfico de un caso importado. Por ejemplo, si el perro que causó la alerta en Toledo en 2013 no hubiese llevado microchip, no se hubiese podido localizar a los propietarios, pero se hubiese podido trazar fácilmente la procedencia marroquí de la cepa a través de la secuencia del virus.

Durante este período se han estudiado en el CNM más de 6.500 casos de animales sospechosos y 26 sospechas de rabia humana, habiéndose diagnosticado 77 casos positivos por RABV en Ceuta y Melilla, el caso canino importado en 2013 en Toledo, los tres casos humanos importados, uno de los cuales recibió un tratamiento experimental que requirió monitorización de marcadores de infección durante semanas y 37 casos de rabia en murciélagos. Estos últimos fueron todos causados por EBLV1, excepto uno correspondiente a un murciélago de cueva (Miniopterus schreibersii) moribundo 
recogido en 2012 en una nave industrial de la ciudad de Lérida y llevado a un centro de recuperación de fauna salvaje donde murió, siendo el cadáver enviado al CNM en el contexto del programa de vigilancia de rabia. Las analíticas mostraron que el animal estaba infectado por un lisavirus desconocido que, tras su caracterización, ha sido admitido por el Comité Internacional de Taxonomía de Virus como un nuevo virus denominado LLEBV (Aréchiga-Ceballos, 2013). La incorporación paulatina de centros de recuperación de fauna salvaje a las actividades de vigilancia de rabia en murciélago, especialmente activa en los de Torreferrussa y Vallcalent en Cataluña, ha supuesto un impulso fundamental para este programa.

\section{Investigación sobre rabia en el Centro Nacional de Microbiología}

Las actividades de investigación sobre rabia en el CNM están ligadas intrínsecamente al programa de vigilancia. Por una parte, la investigación es la base para el desarrollo de la metodología diagnóstica y de caracterización aplicada en la vigilancia y por otra, esta es la fuente de preguntas que alimenta y da sentido a la investigación.

Una de las dos líneas principales de investigación en rabia en el CNM es el estudio de la epidemiología molecular de la rabia en Ceuta y Melilla en el contexto norteafricano. Para ello se formó parte del proyecto europeo RABMEDCONTROL liderado por el Instituto Pasteur con participación de países del sur del Europa y el norte de África. Así, se obtuvo la secuencia de los virus presentes en muestras de Túnez, Argelia y Marruecos, así como de Ceuta y Melilla y se estudiaron los patrones de dispersión del virus mediante un análisis integrado de las secuencias genómicas y una matriz de datos geográficos, mostrando que las fronteras geográficas y la red de carretas son los factores que más influyen en la dispersión del virus, en el norte de África, tal y como como corresponde a un reservorio doméstico como el perro. Las cepas importadas en Ceuta y Melilla eran diferentes entre sí y se correspondían con las circulantes en las regiones marroquíes circundantes a cada ciudad. En ambas ciudades se observan episodios de aparente de reemplazo de cepas que, posiblemente, deben tener que ver con la dinámica del virus en los territorios endémicos circundantes (Talbi, 2010). El estudio en detalle de la dinámica de importación de cepas en ambas ciudades es una línea actualmente en desarrollo.

La línea de investigación de mayor recorrido es, sin duda, la centrada en el estudio de la rabia en murciélagos ibéricos, que se hace en estrecha colaboración con expertos en murciélagos, muy especialmente de la Estación Biológica de Doñana (EBD, CSIC, Sevilla) y de la Universidad de Alcalá de Henares. Los primeros estudios se encaminaron a investigar la dinámica de la infección por EBLV1 en Eptesicus isabellinus de Andalucía, a través la monitorización de colonias naturales mediante captura con red y muestreo de exudados faríngeos para determinar la presencia de genoma vírico por PCR, y de suero para determinación de anticuerpos, al objeto de dar continuidad a la línea iniciada en 1995 por la Estación Biológica de Doñana y el Laboratorio e Salud Público de Málaga con los primeros hallazgos de seroprevalencia elevada de anticuerpos frente a EBLV-1 en colonias de E. isabellinus de Huelva (Pérez Jordá, 1995). Estos trabajos fueron pioneros en demostrar que la infección asintomática o subclínica es un hecho frecuente y que el virus circula de manera endémica en esta especie (Echevarría, 2001; Vázquez-Morón, 2008). Las posibilidades de detectar individuos infectados por este método son muy bajas cuando las colonias a muestrear se eligen al azar, pero aumenta considerablemente cuando nos centramos en colonias cercanas a casos de ejemplares infectados detectados por el sistema de vigilancia. Estos resultados se han corroborado en colonias e Eptesicus serotinus en Alemania (Shatz, 2014) y Francia (Picard-Meyer, 2017; Robardet, 2017). Una importante aportación de nuestros colaboradores de la EBD, fue descubrir que los Eptesicus serotinus de la mitad sur de la Península Ibérica pertenecen, en realidad, a otra especie críptica denominada Eptesicus isabellinus (Ibáñez, 2006) presente en el norte de África. Más aún, existe flujo genético entre las poblaciones de esta especie de ambos lados del Estrecho de Gibraltar (Juste, 2009), lo que sugiere la posibilidad de que EBLV-1 pueda estar presente en el norte de África, aunque hasta el momento no se haya descrito ningún caso, lo cual podría deberse a la escasa vigilancia de rabia en murciélagos en estas áreas. De existir estas poblaciones norteafricanas, su papel en la filogeografía del virus podría ser relevante. 
Otros grupos han realizado investigaciones similares en un rango más amplio de especies en España, describiendo presencia de anticuerpos frente a EBLV-1 en suero y secuencias cortas de ARN viral en pellets sanguíneos y cerebros en un rango amplio de ellas, describiendo, incluso, un modelo de circulación en Myotis myotis, Miniopterus schreibersii y Rinolophus ferrumequinum (Serra-Cobo, 2002, 2013; Amengual 2007; Pons-Salort, 2014). Se han descrito hallazgos similares de presencia de anticuerpos frente a EBLV-1 en un abanico variado de especies en otros países europeos, e incluso de Asia y África. La aparente discrepancia entre los datos de vigilancia pasiva, que nos presentan a EBLV-1 exclusivamente ligado a E. serotinus y E. isabellinus europeos y los datos de detección de anticuerpos, que nos presentan un rango geográfico y de hospedadores amplio, pueden tener varias explicaciones alternativas. Por una parte, E. serotinus y E. isabellinus son especies sinantrópicas que interaccionan frecuentemente con los seres humanos y que, por tanto, son recogidos con mucha frecuencia para su análisis en el laboratorio, mientras que llegan raramente al laboratorio ejemplares de otras especies menos adaptados a hábitats urbanos o semi-urbanos. De hecho, nunca se han recibido ejemplares, o solo de forma muy puntual, de algunas especies con papel conocido como reservorio de lisavirus. Por otra parte, muchas de estas reacciones serológicas podrían estar reflejando cruces antigénicos con otros lisavirus desconocidos. En este sentido, las secuencias de EBLV-1 encontradas en el transcurso de estos estudios han sido detectadas de manera exclusiva por PCR anidada y son muy cortas y poco informativas, lo que impide conocer con precisión la identidad de estos lisavirus. Tampoco se han logrado obtener resultados positivos por detección de antígeno en cerebros de ejemplares de estas especies positivos por PCR anidada, lo cual contrasta con la experiencia extraída de la vigilancia pasiva. Los análisis filogenéticos realizados con estas secuencias cortas, aun siendo poco discriminatorias, muestran un grado de variabilidad dentro de una misma colonia aparentemente similar al que presentan las cepas asociadas a EBLV-1 en toda Europa (Mingo, 2017), lo cual resulta sorprendente. Es imprescindible obtener genomas completos, o al menos secuencias completas de genes completos, de EBLV-1 ligados a especies diferentes de E. serotinus y E. isabellinus que hayan mostrado seroprevalencias significativas, a fin de aclarar el origen y el significado de estas reactividades serológicas. Tras analizar miles de exudados faríngeos de ejemplares de casi todas las especies de murciélagos ibéricos en el CNM, nunca hemos amplificado secuencias de lisavirus en ninguna otra especie aparte de Eptesicus sp.

Otra serie de estudios del CNM se han centrado en la filogeografía del EBLV-1 a nivel europeo e ibérico (Vázquez-Morón, 2011; Mingo, 2018). Existen dos subtipos de EBLV-1, el 1a, muy homogéneo y ampliamente distribuido de este a oeste desde Holanda hasta Rusia y Ucrania, con un subclado disyunto en el sur de Francia y el 1b, exclusivo de Holanda, extremo sur de Alemania y especialmente Francia, donde presenta varios subclados geográficos (Troupin, 2017; Mingo, 2018). En nuestros trabajos hemos encontrado que las cepas ibéricas forman un clado independiente dentro del subtipo $1 \mathrm{~b}$ con cierta aparente estructura geográfica interna. Las cepas ibéricas de subtipo 1a, que hemos encontrado únicamente en las provincias de Huesca y Lérida, agrupan con el clado del sur de Francia y parecen haber entrado recientemente en la Península, estando posiblemente en expansión. Finalmente, tanto los estudios filogenéticos de EBLV-1 (Mingo, 2018), como los de hibridación interespecífica de sus especies reservorias (Centeno, 2019) apuntan a un flujo de dispersión de EBLV1 de sur a norte, por transmisión desde E. isabellinus a E. serotinus. Actualmente se están haciendo estudios con genoma completo a fin de afinar la estructura poblacional de EBLV-1b a nivel ibérico y de buscar signos de adaptación a especie, ya que tenemos cepas de virus asociadas a las dos especies crípticas presentes en la Península Ibérica (E. serotinus, E. isabellinus). Los estudios de co-evolución entre EBLV-1 y E. serotinus a nivel europeo han arrojado resultados negativos (Troupin, 2017), indicando que la filogeografía del virus responde a causas diferentes. Actualmente tenemos en curso estudios similares para el modelo EBLV-1/E. isabellinus en la Península Ibérica.

Finalmente, el descubrimiento del LLEBV (Aréchiga, 2013) nos llevó a avanzar en su caracterización en colaboración con la Agencia de Laboratorios Veterinarios del Reino Unido, habiendo obtenido en primera instancia su genoma completo (Martson, 2016), lo que permitió corroborar las hipótesis originales basadas en la secuencia del gen de la proteína de nucleocápside, que mostraba a LLEBV como un lisavirus divergente con respecto al filogrupo 1, que es el que engloba 
a RABV y al resto de los lisavirus neutralizables por anticuerpos inducidos por vacunación. En un segundo trabajo se corroboró que LLEBV no es neutralizado por anticuerpos inducidos por vacuna por lo que, de ser un virus transmisible a los seres humanos, careceríamos de herramientas para su control (Banyard, 2018). La presencia en Europa de lisavirus divergentes como LLEBV o WCBV se considera un potencial problema de salud pública (Echevarría, 2019).

\section{Investigaciones de virus potencialmente emergentes asociados a murciélagos ibéricos en el Centro nacional de Microbiología}

La experiencia de monitorización de colonias e murciélago para los trabajos de infección por lisavirus, nos permitió ampliar nuestro ámbito a otras familias de virus patógenos relacionadas con murciélagos a través de la participación de laboratorios del CNM especializados en otros grupos de virus, como el de Virus Respiratorios y el de Enfermedades Importadas y Arbovirus.

En el contexto de estos estudios se descubrió el primer filovirus europeo, el virus Lloviu (LLOV), asociado a un episodio internacional de mortandad masiva de murciélago de cueva (Miniopterus schreibersii) (Negredo, 2011). Este virus ha ido aceptado por el ICTV como único miembro de un nuevo género denominado Cuevavirus dentro de la familia Filoviridae, independiente de los géneros Marburgvirus y Ebolavirus pero más relacionado con este último. Su relación con el episodio internacional de alta mortalidad ocurrido en el año 2002 nos hizo pensar que esta especie no fuese su reservorio natural, sin embargo, estudios más recientes (Ramírez de Arellano, 2019) nos han permitido comprobar, en las mismas poblaciones originalmente afectadas, la presencia de anticuerpos en individuos jóvenes sanos de esta misma especie nacidos bastantes años después del episodio de mortalidad, sugiriendo que actualmente existe circulación de este virus o de algún otro antigénicamente relacionado sin producir mortandad, lo cual es necesario continuar investigando.

En un estudio de búsqueda de coronavirus pudimos encontrar secuencias de alfa y beta coronavirus en varias especies e murciélago ibéricos, una de ellas relativamente cercana a MERS-CoV (Falcón, 2011). No se encontraron coronavirus tipo SARS, aunque la muestra de murciélagos de herradura (gen. Rhinolophus), que son los que habitualmente se asocian a este grupo de virus, no fue lo suficientemente representativa como para excluir su presencia, más aún cuando han sido descritos en otros países europeos. Actualmente estamos ampliando la búsqueda de coronavirus tipo SARS en murciélagos de herradura ibéricos (figura 3).

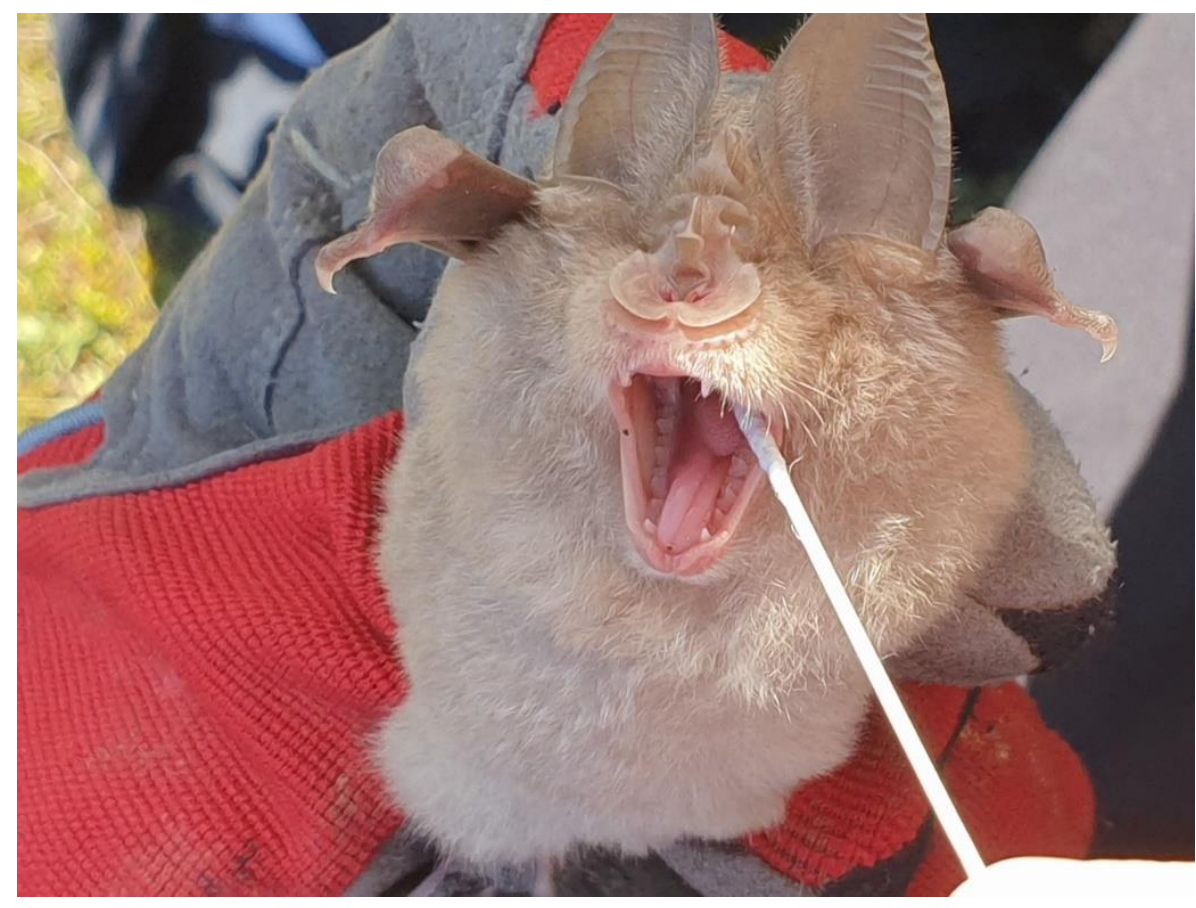

Figura 3 Toma de exudado orofaríngeo a un murciélago de herradura. 
Finalmente, otra línea de trabajo se centra en la relación virus-huésped en murciélagos ibéricos y más concretamente en la búsqueda de signos de evolución paralela. Para ello se han estudiado dos modelos de virus DNA, los adenovirus y los herpesvirus. Estos últimos son muy prevalentes y casi un $30 \%$ de los individuos analizados mostraban presencia de genomas de un betaherpesvirus asimilable al citomegalovirus humano que formaba un único clado independiente asociado a murciélagos. Los grupos encontrados dentro de este clado remedan la filogenia de los murciélagos asociados, lo cual es un claro indicio de evolución paralela (Pozo, 2016). Los estudios con adenovirus han mostrado igualmente signos de co-evolución virus-huésped (Iglesias, 2018), siendo las estrategias de apareamiento un factor determinante en la dispersión de estos virus (Rossetto, 2020). Actualmente estamos estudiando los herpesvirus de murciélago asociados a dos complejos diferentes de especies crípticas ibéricas.

Finalmente, otras colaboraciones más puntuales con otros grupos nos han llevado a participar en la descripción de un complejo de papilomavirus en murciélagos (García-Pérez, 2014) y de bacterias del género Bartonella (Stuckey, 2017).

\section{Ningún caso de rabia humana transmitida por perros en 2030, un reto posible}

Quiero terminar aludiendo a lo que debe ser el norte de la comunidad científica dedicada al estudio de la rabia: el gran objetivo global de la eliminación de la rabia humana. La UE ha logrado, no solo la eliminación de la rabia humana y canina, sino incluso la de la rabia salvaje vulpina, demostrando que el objetivo global de acabar con los casos de rabia humana transmitidos por perros en 2030 no supone ningún problema de tipo técnico, sino únicamente económico y logístico. El ingente despliegue global de medios dispuesto para el control de la actual pandemia de COVID-19 nos está mostrando con meridiana claridad que este tipo de retos son esencialmente una cuestión de voluntad política. Su magnitud es de tal envergadura que en el futuro va a ser muy difícil argumentar que el control de las enfermedades olvidadas está fuera del alcance de esa Comunidad Internacional a la que tanto nos cuesta llamar, simplemente, Humanidad.3. Resultados

\section{Referencias Bibliográficas}

1. Amengual B, Bourhy H, López-Roig M, Serra-Cobo J. Temporal dynamics of European bat Lyssavirus type 1 and survival of Myotis myotis bats in natural colonies. PLoS One, 2007,27;2(6): e566.

2. Aréchiga-Ceballos N, Vázquez-Morón S, Berciano JM, Nicolás O, Aznar- López C, Juste J, RodríguezNevado C, Aguilar-Setién A, Echevarría JE. Novel Lyssavirus in Spain. Emerging infectious Diseases, 2013,19(5): 793-795.

3. Banyard AC, Selden D, Wu G; Thorne L, Jennings D, Marston D, Finke S, Freuling CM, Mueller T, Echevarria JE, Fooks AR. Isolation, antigenicity and immunogenicity of Lleida Bat Lyssavirus. Journal of General Virology, 2018, 99(12):1590-1599

4. Centeno-Cuadrosa A, Razgourb O, García-Mudarrac JL, Mingo-Casas P, Sandonís V, Redondo A, Ibáñez C, de Paz O, Martinez-Alós S, Pérez Suarez G, Echevarría JE, Juste J. Comparative phylogeography and asymmetric hybridization between cryptic bat species. Journal of Zoological Systematics and Evolutionary Research, 2019.

5. Dacheux L, Larrous F, Mailles A, Boisseleau D, Delmas O, Biron C, Bouchier C, Capek I, Muller M, Ilari F, Lefranc T, Raffi F, Goudal M, Bourhy H. European bat Lyssavirus transmission among cats, Europe. Emerg Infect Dis. 2009.15(2):280-4

6. Echevarría JE, Avellón A, Juste J, Vera M, Ibáñez C. Screening of active lyssavirus infection in wild bat populations by viral RNA on oropharyngeal swabs. Journal of Clinical Microbiology, 2001, 39(10): 36783683.

7. Echevarría JE, Banyard AC, McElhinney LM, Fooks AR. Current Rabies Vaccines Do Not Confer Protective Immunity against Divergent Lyssaviruses Circulating in Europe. Viruses, 2019, 11, 892;

8. Fahrion AS, Mikhailov A, Abela-Ridder B, Giacinti J, Fahrlon JH, Human rabies transmitted by dogs: current status of global data, 2015. Weekly Epidemiological Record, 2016, 91: 13-20. 
9. Falcón A, Vázquez-Morón S; Casas I Aznar C, Ruiz G, Pozo F, Pérez-Breña P, Juste J, Ibáñez C, Garin I, Aihartza J, Echevarría JE. Detection of alpha and betacoronaviruses in multiple iberian bat species. Archives of Virology, 2011, 156:1883-1890.

10. García-Pérez R, Ibáñez C, Godínez JM, Aréchiga N, Garin I, Pérez-Suárez G, de Paz O, Juste J, Echevarría JE, Bravo IG. Novel papillomaviruses in free-ranging Iberian bats: no virus-host co-evolution, no strict host specificity and hints for recombination. Genome Biology and Evolution, 2014, 6(1): 94-104.

11. Gautret P, Ribadeau-Dumas F, Parola P, Brouqui P, Bourhy H. Risk for rabies importation from North Africa. Emerging Infectious Diseases, 2011, 17(12):2187-93.

12. Gossner CM, Mailles A, Aznar I, Dimina E, Echevarría JE, Feruglio SL, Lange H, Maraglino FP, Parodi P, Perevoscikovs J, Van der Stede Y, Bakonyi T. Prevention of human rabies: a challenge for the European Union and the European Economic Area. Eurosurveillance, 2020, 25(38):pii=2000158.

13. Hampson K, Coudeville L, Lembo T, Sambo M, Kieffer A, Attlan M, Barrat J, Blanton JD, Briggs DJ, Cleaveland S, Costa P, Freuling CM, Hiby E, Knopf L, Leanes F, Meslin FX, Metlin A, Miranda ME, Müller T, Nel LH, Recuenco S, Rupprecht CE, Schumacher C, Taylor L, Vigilato MA, Zinsstag J, Dushoff J; Global Alliance for Rabies Control Partners for Rabies Prevention. Estimating the global burden of endemic canine rabies. PLoS Neglected Tropical Diseases, 2015, 16;9(4):e0003709. Erratum in:PLoS Neglected Tropical Diseases, 2015,9(5):e0003786.

14. Hoffmann B, Freuling CM, Wakeley PR, Rasmussen TB, Leech S, et alImproved safety for molecular diagnosis of classical rabies viruses by use of a TaqMan realNEGtime reverse transcriptionNEGPCR “double check" strategy. Journal of Clinical Microbiology, 2010, 48(11): 3970-8),

15. Ibáñez, C., García-Mudarra, J. L., Ruedi, M., Stadelmann, B. \& Juste, J. The Iberian contribution to cryptic diversity in European bats. Acta Chiropterologica, 2006, 8, 277-297.

16. Iglesias-Caballero M, Juste J, Vázquez-Morón S, Falcon A, Aznar-Lopez C, Ibáñez C, Pozo F, Ruiz G, Berciano JM, Garin I, Aihartza J, Echevarría JE, Casas I. New Adenovirus Groups in Western Palaearctic Bats. Viruses, 2018, 20;10(8)

17. Johnson N, Vos A, Freuling C, Tordo N, Fooks AR, Müller T. Human rabies due to lyssavirus infection of bat origin. Veterinary Microbiology, 2010, 19;142(3-4):151

18. Juste J, Bilgin R, Muñoz J, Ibáñez C. Mitochondrial DNA signatures at different spatial scales: from the effects of the Straits of Gibraltar to population structure in the meridional serotine bat (Eptesicus isabellinus). Heredity, 2009, 103, 178-187

19. King AA, Fooks AR, Aubert M, Wandeler, Eds. AI. Historical perspective of rabies in Europe and the Mediterranean Basin. OIE, 2004, París.

20. Mailles A, Boisseleau D, Dacheux L, Michalewiscz C, Gloaguen C, Ponçon N, Bourhy H, Callon H, Vaillant V, Dabosville I, Morineau-Le Houssine P. Rabid dog illegally imported to France from Morocco, August 2011. Euro Surveillance, 2011, 18;16(33):19946.

21. Marston DA, Ellis RJ,. Wise EL, Aréchiga-Ceballos N,Freuling CM, Banyard AC, McElhinney LM, Lamballerie X, Müller T, Fooks AR, Echevarría JE. Complete Genomic Sequence of Lleida Bat Lyssavirus. Genome Announcements, 2016, 5(2): e01427-16.

22. Mingo-Casas P, Sandonís V, Vázquez-Morón S, Berciano JM, Juste J, Echevarría JE Rabies in Spain. A Peculiarity in Eurasia. Annals of Virology and Research, 2017, 3(2): 1030.

23. Mingo-Casas P, Sandonís V, Obón E, Berciano JM, Vázquez-Morón S, Juste J, Echevarría JE. First cases of European Bat Lyssavirus type 1 in Iberian serotine bats: implications for the molecular epidemiology of bat rabies in Europe. Plos Neglected Tropical Diseases, 2018,12(4): e0006290

24. Ministerio de Agricultura, Alimentación y Medio Ambiente, Ministerio de Sanidad, Servicios Sociales e Igualdad, Ministerio de Economía y Competitividad. Protocolo de Actuación ante mordeduras de animales. Madrid:

2013. https://www.mscbs.gob.es/profesionales/saludPublica/sanidadExterior/docs/protocoloActuacion_morde duras_agresiones_animales_Junio2013.pdf

25. Müller T, Cox J, Peter W, Schäfer R, Johnson N, McElhinney LM, Geue JL, Tjørnehøj K, Fooks AR. Spillover of European bat lyssavirus type 1 into a stone marten (Martes foina) in Germany. Journal of Veterinary Medicine, Series B, 2004, 51(2):49-54.

26. Nathwani D, McIntyre PG, White K, Shearer AJ, Reynolds N, Walker D, Orange GV, Fooks AR. Fatal human rabies caused by European bat Lyssavirus type 2a infection in Scotland. Clinical Infectious Diseases, 2003,15;37(4):598-601. 
27. Negredo A, Palacios G, Vázquez-Morón S, González F, Dopazo H, Molero F, Juste J, Quetglas J, Savji N, Martínez MC, Herrera JE, Pizarro M, Hutchison S, Echevarría JE, Lipkin IW, Tenorio A. Discovery of an ebolavirus-like filovirus in Europe. Plos Pathogens. 2011, 7(10)

28. Organización Mundial de la sanidad Animal (OIE). Manual de las Pruebas de Diagnóstico y de las Vacunas para los Animales Terrestres 2019. https:/www.oie.int/es/que-hacemos/normas/codigos-ymanuales/acceso-en-linea-al-manual-terrestre/

29. Perez de Diego AC, Vigo M, Monsalve J, Escudero A. The One Health approach for the management of an imported case of rabies in mainland Spain in 2013. Euro Surveillance, 2015, 12;20(6):21033.

30. Pérez-Jordá JL, Ibáñez C, Muñoz-Cervera M, Téllez A. Lyssavirus en Eptesicus serotinus (Chiroptera, vespertilionidae). Journal of Wildlife Diseases, 1995, 31 (3):372-377.

31. Picard-Meyer E, Barrat J, Wasniewski M, Wandeler A, Nadin-Navis S, Lowings JP, Fooks A, Mchelhinney L, Bruyère V, Cliquet F. Epidemiology of rabid bats in France, 1989 to 2002. Veterinary record, 2004, 155: 774-777.

32. Picard-Meyer E, Servat A, Wasniewski M, Gaillard M, Borel C, Cliquet F. Bat rabies surveillance in France: first report of unusual mortality among serotine bats. BMC Veterinary Research, 2017, 13;13(1):387

33. Pons-Salort M, Serra-Cobo J, Jay F, López-Roig M, Lavenir R, Guillemot D, Letort V, Bourhy H, Opatowski L. Insights into persistence mechanisms of a zoonotic virus in bat colonies using a multispecies metapopulation model. PLoS One, 2014,22;9(4):e95610.

34. Pozo F, Juste J, Vázquez-Morón S., Aznar-López C, Ibáñez C, Garin I, Aihartza J, Casa I, Tenorio A, Echevarría JE. Identification of novel Betaherpesviruses in iberian bats reveals parallel evolution. PLoS ONE, 2016, 11(12): e0169153.

35. Ramirez de Arellano E, Sánchez-Lockhart M, Perteguer MJ, Bartlett M, Ortiz M, Campioli P, Hernández A, Gonzalez J, Garcia C, Ramos M, Jimenez MA, Tenorio A, Sánchez-Seco MP, González F, Echevarria JE, Palacios G, Negredo A. First evidence of LLOV antibodies in Schreiber's Bent-winged insectivorous bats demonstrate a wide circulation of the virus in Spain. Viruses, 2019, 11, 360;

36. Regnault B, Evrard B, Plu I, Dacheux L, Troadec E, Cozette P, Chrétien D, Duchesne M, Jean-Michel V, Jamet A, Leruez M, Pérot P, Bourhy H, Eloit M, Seilhean D. First case of lethal encephalitis in Western Europe due to European bat lyssavirus type 1. Clinical Infectious Diseases, 2021, 15:443

37. Robardet E, Borel C, Moinet M, Jouan D, Wasniewski M, Barrat J, Boué F, Montchâtre-Leroy E, Servat A, Gimenez O, Cliquet F, Picard-Meyer E. Longitudinal survey of two serotine bat (Eptesicus serotinus) maternity colonies exposed to EBLV-1 (European Bat Lyssavirus type 1): Assessment of survival and serological status variations using capture-recapture models. PLoS Neglected Tropical Diseases, 2017, 17;11(11):e0006048.

38. Rossetto F, Iglesias-Caballero M, Liedtke HC, Gomez-Mestre I, Berciano JM, Pérez-Suárez G, de Paz O, Ibáñez C, Echevarría JE, Casas I, Juste J. Mating strategy is determinant of adenovirus prevalence in European bats. PLoS One, 2020, 7;15(1):e0226203.

39. Ruppretch CE, Fooks AR, Abela-Ridder B. Eds. Laboratory techniques in rabies, fifth edition. Geneva: World Health Organization (WHO); 2018.

40. Schatz J, Fooks AR, McElhinney L, Horton D, Echevarria J, Vázquez-Moron S, Kooi EA, Rasmussen TB, Müller T, Freuling CM. Bat Rabies Surveillance in Europe. Zoonoses and Public Health, 2013, 60, $22-34$. .

41. Schatz J, Ohlendorf B, Busse P, Pelz G, Dolch D, Teubner J, Encarnação JA, Mühle RU, Fischer M, Hoffmann B, Kwasnitschka L, Balkema-Buschmann A, Mettenleiter TC, Müller T, Freuling CM. Twenty years of active bat rabies surveillance in Germany: a detailed analysis and future perspectives. Epidemiology and Infection, 2014,142(6):1155-66.

42. Serra-Cobo J, Amengual B, Abellán C, Bourhy H. European bat lyssavirus infection in Spanish bat populations. Emerging Infectious Diseases, 2002,8(4):413-20.

43. Serra-Cobo J, López-Roig M, Seguí M, Sánchez LP, Nadal J, Borrás M, Lavenir R, Bourhy H. Ecological factors associated with European bat lyssavirus seroprevalence in spanish bats. PLoS One, 2013, 20;8(5):e64467.

44. Strauss R, Granz A, Wassermann-Neuhold M, Krause R, Bago Z, Revilla-Fernandez S, Simón-Soria F, Echevarría JE, Popow-Kraupp T, Allerberger F, Schonbauer M, Hrabcik H. A human case of travel-related rabies in Austria, September 2004. Eurosurveillance, 2005, 10(11):225-226. 
45. Stuckey MJ, Boulouis HJ, Cliquet F, Picard-Meyer E, Servat A, Aréchiga-Ceballos N, Echevarría JE, Chomel BB. Potentially zoonotic bartonella in bats from France and Spain. Emerging Infectious Diseases, 2017, 23(3): 539-541.

46. Suárez-Rodríguez B, Santos S, Saravia G, Sánchez-Gómez A, Sierra MJ, Amela C, Gutierrez-Avila G, Jané M, Canales AJ, Ripalda J, Lopaz MA, Sáez JL, García-Villacieros E, Echevarría JE, Vázquez S, RodríguezValín E, Simón F. A case of rabies in a dog imported to Spain from Morocco in June 2013. Temporary loss of rabies free Certificate. Rabies Bulletin Europe, 2013, 37(2): 5-9

47. Talbi C, Lemey P, Suchard MA, Abdelatif E, Elharrak M, Jalal N, Faouzi A, Echevarría JE, Vazquez Morón S, Rambaut A, Campiz N, Tatem AJ, Holmes EC, Bourhy H. Phylodynamics and human-mediated dispersal of a zoonotic virus. PLoS Pathogens, 2010,28;6(10):e1001166.

48. Tjørnehøj K, Fooks AR, Agerholm JS, Rønsholt L. Natural and experimental infection of sheep with European bat lyssavirus type-1 of Danish bat origin. Journal Comparative Pathology, 2006, 134(2-3):190201.

49. Troupin C, Picard-Meyer E, Dellicour S, Casademont I, Kergoat L, Lepelletier A, Dacheux L, Baele G, Monchâtre-Leroy E, Cliquet F, Lemey P, Bourhy H. Host Genetic Variation Does Not Determine SpatioTemporal Patterns of European Bat 1 Lyssavirus. Genome Biology and Evolution, 2017, 9(11):3202-3213.

50. Vázquez S, Avellón A, Echevarría JE RT-PCR for detection of all seven genotypes of Lyssavirus genus. Journal of Virological Methods, 2006, 135: 281-287.

51. Vázquez-Morón S, Juste J, Ibáñez C, Ruiz-Villamor R, Avellón A, Vera M, Echevarría JE Endemic circulation of European bat lyssavirus type 1 in serotine bats, Spain. Emerging Infectious Diseases, 2008, 14(8): 1263-1266.

52. Vázquez-Morón S, Juste J, Ibáñez C, Berciano JM, Echevarría JE Phylogenetic Characterization of European Bat Lyssavirus Type 1 in Eptesicus isabellinus, Spain. Emerging Infectious Diseases, 2011, 17 (3): 520-23.

53. World Health Organization (WHO), Food and Agriculture Organization of the United Nations (FAO) and World Organisation for Animal Health (OIE). Zero by 30: the global strategic plan to end human deaths from dog-mediated rabies by 2030. WHO, FAO, OIE, 2018. 